\title{
Diabetes and correlates of cardiovascular diseases among women aged 15-49 years in Benin: evidence from a Demographic and Health Survey
}

\section{Michael Ekholuenetale}

University of Ibadan

Chimezie Igwegbe Nzoputam

University of Benin

Amadou Barrow ( $\sim$ abarrow@utg.edu.gm )

University of The Gambia https://orcid.org/0000-0002-6006-9355

Original investigation

Keywords: High blood pressure, High blood sugar, Diabetes, Heart disease, Lung disease

Posted Date: August 19th, 2020

DOI: https://doi.org/10.21203/rs.3.rs-51774/v1

License: (c) (i) This work is licensed under a Creative Commons Attribution 4.0 International License.

Read Full License 


\section{Abstract}

Background: Sub-Saharan Africa (SSA) countries are facing an epidemiological shift from infectious diseases to chronic diseases, such as cardiovascular diseases (CVDs). The burden of CVDs in a population results from the prevalence of several factors. This study was to determine the association of diabetes and correlates with heart and lung diseases.

Methods: We used Benin Demographic and Health Survey (BDHS) population-based cross-sectional data. BDHS 2017-18 is the fifth of its kind. A total of 7712 women of reproductive age were included in this study. Heart and lung diseases were the outcome variables. Percentage and logistic regression model were used to analyze the data. The level of statistical significance was set at $5 \%$.

Results: The prevalence of heart disease was 1.3\% (95\% Cl: 1.0\%-1.7\%) and lung disease was approximately $1.5 \%$ (95\% Cl: $1.2 \%-1.9 \%)$. Women who had diabetes were also found to be 3.57 times significantly more likely to have heart disease when compared with those who do not have diabetes $(A O R=3.57 ; 95 \% \mathrm{Cl}: 1.51-8.45)$. Furthermore, women with diabetes were 4.55 times significantly more likely to also have lung diseases when compared with those who do not have diabetes (AOR= 4.55; $95 \% \mathrm{Cl}$ : 2.06 - 10.06). Women who had hypertension were found to be 3.18 times significantly more likely to have heart disease when compared with those who had no hypertension (adjusted odds ratio $(A O R)=$ $3.18 ; 95 \% \mathrm{Cl}: 2.02-4.98)$.

Conclusion: Diabetes was a key determinant of heart disease. Health policymakers and government need to focus on widespread prevention and control interventions of heart disease through improved screening for risk factors and early detection of CVDs especially among high-risk women. The government should also create nationwide awareness and educate people about CVDs and its fatality rate.

\section{Background}

Non-communicable diseases (NCDs) have been associated with great deal of deaths globally, with Disability-Adjusted Life-Years (DALYs) increase of $67.0 \%$ between 1990 and 2017. Heart and lungs related diseases share an enormous part of these deaths (1). They constitute the larger percentage $(63 \%)$ of the deaths globally, with over 36 million people dying of the diseases on annual basis. Countries with low and middle income, such as Benin and other SSA countries, have been observed to be contributing $86 \%$ of these untimely deaths (2). It is also reported that $39 \%$ of these deaths occur among people of age $30-$ 70 years. It has been projected that if this trend continues unchecked, it will result to revenue loss to the tune of US\$7 trillion, in the next decade and also plunge millions of people into abject poverty (2).

On a global level, deaths as a result of heart-related diseases witnessed a $21.1 \%$ increase between 2007 and 2017 according to the Global Burden of Diseases (GBDs) report 2017 (4). The report also stated that rate of death had a negative trend as there was a decrease from 259.9 observed in 2007 to 233.1/100,000 deaths recorded in 2017 (4). Heart disease is the second leading cause of noncommunicable disease burden in SSA, accounting for a total of 22.9 million Disability-Adjusted Life- 
Years. This is posing an enormous challenge to the SSA health systems (3). Stroke and ischemic heart disease, together, contributed a chunk (84.9\%) of all these cardiovascular-related deaths, increasing from 5.29 million and 7.30 million in 2007 to 6.17 million and 8.93 million deaths in 2017, respectively (4). Lower respiratory infections, stroke, ischemic heart disease with chronic obstructive pulmonary disease (COPD) were among the five leading non-communicable diseases that caused Disability-Adjusted Life Years (DALYs) at the global level in 2017 with an increase of $40.1 \%$ (5).

The burden of lung disease is currently a growing concern in SSA (6). This can be attributed to the recent increase in data concerning a good number of risk factors for lung diseases such as the use of tobacco and its related products (7), exposure to industrial and vehicular emissions as well as other outdoor air pollution (8). Unfortunately, data on the estimates of complications and deaths as a result of lung diseases in most SSA countries are often not available as routine vital registrations are habitually not available $(9,10)$. However, in a project conducted in Benin on hearts-related NCDs shows that risk factors for CVDs such as high blood pressure and obesity were $35.5 \%$ and $10.7 \%$ respectively, among the studied population, with crude mortality rate of 12.5 for every 1,000 person-year (12). A comprehensive knowledge and understanding of these trends in changes in global health status and the foremost causes of burden of diseases is critical to the achievement of the third Sustainable Development Goal (SDG-3) (11).

Regardless of this burden, the sub-region is now witnessing a rapid epidemiological evolution which is characterized by a move from dominance of communicable diseases to non-communicable ones, just as its being observed in many other low and middle-income economies around the globe (13). Nevertheless, there is a growing research indicating that disease conditions such as diabetes $(14,15)$ lung-related and respiratory diseases $(16)$, and other CVDs $(17,18)$, have now become serious disease-burden in many SSA countries. Risk factors for lung and heart diseases' surveillance in SSA for the past three to four decades show that majority of young adults are exposed to one or more NCDs risk factors. These risk factors include tobacco and excessive alcohol use, poor diet, sedentary lifestyle, hypertension as well as overweight or obesity (19).

In the SDGs, the challenges of the NCDs across the globe were put into consideration. The document targets the reduction by $30 \%$ by the year 2030 , including all premature deaths caused by the foremost NCDs such as lungs and heart-related diseases. It also targets the promotion of general wellbeing and mental health, as enshrined in the SDGs goal $3(11,20)$. This is also one of the targets of the Global NCDs Action Plan 2013-2020 (21). The achievement of these targets by any of the countries in SSA, such as Benin Republic, will be dependent on a total overhauling of the health systems by making sure all the bottlenecks such as underfunding, poor and inadequate training and poor equipment of the health sector, are arrested. The majority of the health systems in SSA are fragmented, under-funded, fragile and infrastructurally limited to handle this ever-increasing communicable and non-communicable disease burden $(22,23)$. In light of the above, we undertook this study to examine the association between diabetes and correlates of cardiovascular diseases among women aged 15-49 years in Benin. 


\section{Methods}

\section{Data source}

We used BDHS population-based cross-sectional data. BDHS 2017-18 is the fifth of its kind. A total of 7712 women of reproductive age were included in this study. BDHS used a stratified multi-stage cluster random sampling technique for the data collection. Data was collected on vital reproductive health issues via structured interviewer-administered questionnaires. DHS program was established by the United States Agency for International Development (USAID) in 1984. It was designed as a follow-up to the World Fertility Survey and the Contraceptive Prevalence Survey projects. It was first awarded in 1984 to Westinghouse Health Systems (which subsequently evolved into part of OCR Macro). The project has been implemented in overlapping five-year phases; DHS-I ran from 1984 to1990; DHS-II from 1988 to1993; and DHS-III from 1992 to1998. In 1997, DHS was folded into the new multi-project MEASURE program as MEASURE DHS+. Since 1984, more than 130 nationally representative household-based surveys have been completed under the DHS project in about 70 countries.

Many of the countries have conducted multiple DHS to establish trend data that enable them to gauge progress in their programs. Countries that participate in the DHS program are primarily countries that receive USAID assistance; however, several non-USAID supported countries have participated with funding from other donors such as UNICEF, UNFPA or the World Bank. DHS are designed to collect data on fertility and reproductive health, child health, family planning and HIV/AIDS. Due to the subject matter of the survey, women of reproductive age (15-49) are the main focus of the survey. Women eligible for an individual interview are identified through the households selected in the sample. Therefore, all DHS surveys utilize a minimum of two questionnaires-a Household Questionnaire and a Women's Questionnaire. DHS data is publicly available and can be accessed from the MEASURE DHS database at http://dhsprogram.com/data/available-datasets.cfm. DHS are usually implemented by the National Population Commission (NPC) with financial and technical assistance by ICF International provisioned through the USAID-funded MEASURE DHS program. DHS involved multi-stage stratified cluster design based on a list of enumeration areas (EAs), which are systematically selected units from localities and constitute the Local Government Areas (LGAs). Details of the sampling procedure have been reported previously (24).

\section{Geography of Benin}

The country spans from north to south and indeed a long stretched country in West Africa, which is located west of Nigeria and east of Togo, it is bordered to the north by Niger and Burkina Faso, in the south by the Bight of Benin, the Gulf of Guinea, that part of the tropical North Atlantic Ocean which is roughly south of West Africa. Benin has geographical regions which are; Alibori, Atacora, Atlantique, Borgou, Collines, Couffo, Donga, Littoral, Mono, Quémé, Plateau and Zou. Her coastline is approximately $121 \mathrm{~km}$ long, with an area of $112,622 \mathrm{~km}^{2}$ and has a population of approximately 11.48 million people (in 2018) (25), Porto-Novo, a port on an inlet of the Gulf of Guinea is the nation's capital city, largest city and economic capital is Cotonou. Spoken languages are French (official), Fon and Yoruba (26). 


\section{Selection and measurement of variables}

\section{Outcome variables}

The chronic diseases were measured dichotomously (yes vs. no) as reported by the women; "In the past 12 months, ever told has heart disease" and "ever diagnosed with lung disease by doctor or nurse".

\section{Explanatory variables}

Hypertension and diabetes were measure dichotomously (yes vs. no); usage of tobacco products/cigarette (use vs. not use); ever used anything to delay getting pregnant (yes vs. no); parity: 12/3-4/>4/no birth; total lifetime number of sex partners: $1 / 2 / 3 />3$; religion: Christianity/Islam/Traditional and others; maternal age: 15-19/20-24/25-29/30-34/35-39/40-44/45-49; region: Alibori/Atacora/Atlantique/Borgou/Collines/Couffo/Donga/Littoral/Mono/ Quémé/Plateau and Zou; place of residence: urban/rural; marital status: not married/currently married or living with a partner/formerly married; maternal education: no formal education/primary/secondary+; participation in the labour force: working vs. not working; covered by health insurance: covered vs. not covered. Household wealth quintile: principal components analysis (PCA) was used to assign the wealth indicator weights. This procedure assigned scores and standardized the wealth indicator variables such as; bicycle, motorcycle/scooter, car/truck, main floor material, main wall material, main roof material, sanitation facilities, water source, radio, television, electricity, refrigerator, cooking fuel, furniture, number of persons per room. The factor coefficient scores (factor loadings) and z-scores were calculated. For each household, the indicator values were multiplied by the loadings and summed to produce the household's wealth index value. The standardized z-score was used to disentangle the overall assigned scores to the poor/middle/rich categories $(27,28)$.

\section{Ethical consideration}

We utilized population-based secondary datasets available in the public domain/ online with all identifier information removed. The authors were granted access to use the data by MEASURE DHS/ICF International. DHS Program is consistent with the standards for ensuring the protection of respondents' privacy. ICF International ensures that the survey complies with the U.S. Department of Health and Human Services regulations for the respect of human subjects. No further approval was required for this study. More details about data and ethical standards are available at http://goo.gl/ny8T6X.

\section{Analytical approach}

We used the built-in survey command of Stata for all analyses to account for the sampling strata, primary sampling unit, and sampling weight provided in the dataset. Prevalence of the heart and lung diseases were reported in percentages. The correlation matrix was used to conduct multicollinearity diagnostics to examine the interdependence between explanatory variables using a cut-off minimum of 0.8 known to cause concerns in multicollinearity (29). All significant variables in the unadjusted logistic regression model were retained in the model due to lack of collinearity. Multivariable binary logistic regression was 
used to analyze the data. The level of statistical significance was set at $5 \%$. All data analyses were conducted using Stata 14.0 (StataCorp, College Station, Texas, United States of America).

\section{Results}

The prevalence of heart disease was 1.3\% (95\% Cl: 1.0\%-1.7\%). Results from Table 2 showed $4.7 \%$ and $8.4 \%$ of women, who had hypertension and diabetes respectively, also had heart diseases, in contrast to $1.0 \%$ and $1.2 \%$ of those who do not have hypertension and diabetes but have heart disease respectively. Women who had hypertension were found to be 3.18 times more likely to have heart diseases when compared with those who had no hypertension (AOR $=3.1895 \% \mathrm{Cl}$ : 2.02-4.98). Those who had diabetes were also found to be 3.57 times more likely to have heart disease when compared with those who do not have diabetes $(A O R=3.57 ; 95 \% \mathrm{Cl}: 1.51-8.45)$. Furthermore, women aged $30-34$ years were 3.49 times as likely to have heart disease when compared with women aged $15-19$ years (AOR $=3.49 ; 95 \% \mathrm{Cl}: 1.18$, 10.31). The geographical region was also significantly associated with heart disease (See Table 1 for the details). 
Table 1

Association between diabetes and correlates with heart disease among women aged 15-49 years in Benin

\begin{tabular}{|c|c|c|c|c|c|}
\hline Variable & $\begin{array}{l}\text { Number } \\
\text { of } \\
\text { women } \\
(\%)\end{array}$ & $\begin{array}{l}\text { Number of } \\
\text { women with } \\
\text { heart disease }\end{array}$ & $\begin{array}{l}\text { Percentage (\%) of } \\
\text { women with heart } \\
\text { disease }\end{array}$ & $\begin{array}{l}\text { Unadjusted } \\
\text { OR (95\%Cl) }\end{array}$ & $\begin{array}{l}\text { Adjusted } \\
\text { OR } \\
(95 \% \mathrm{Cl})\end{array}$ \\
\hline \multicolumn{6}{|c|}{ Hypertension } \\
\hline Yes & 751 (9.7) & 35 & 4.7 & $\begin{array}{l}5.11 \\
(3.37- \\
7.75)^{\star}\end{array}$ & $\begin{array}{l}3.18 \\
(2.02- \\
4.98)^{\star}\end{array}$ \\
\hline No & $\begin{array}{l}6961 \\
(90.3)\end{array}$ & 66 & 1.0 & 1.00 & 1.00 \\
\hline \multicolumn{6}{|l|}{ Diabetes } \\
\hline Yes & $83(1.1)$ & 7 & 8.4 & $\begin{array}{l}7.38 \\
(3.32- \\
16.44)^{\star}\end{array}$ & $\begin{array}{l}3.57 \\
(1.51- \\
8.45)^{\star}\end{array}$ \\
\hline No & $\begin{array}{l}7629 \\
(98.9)\end{array}$ & 94 & 1.2 & 1.00 & 1.00 \\
\hline \multicolumn{6}{|c|}{$\begin{array}{l}\text { Usage of } \\
\text { cigarettes or } \\
\text { tobacco products }\end{array}$} \\
\hline Use & $216(2.8)$ & 4 & 1.9 & $\begin{array}{l}0.69 \\
(0.25- \\
1.91)\end{array}$ & \\
\hline Not use & $\begin{array}{l}7496 \\
(97.2)\end{array}$ & 97 & 1.3 & 1.00 & \\
\hline \multicolumn{6}{|c|}{$\begin{array}{l}\text { Ever used any } \\
\text { method to delay } \\
\text { pregnancy }\end{array}$} \\
\hline Yes & $\begin{array}{l}2371 \\
(30.7)\end{array}$ & 42 & 1.8 & $\begin{array}{l}1.61 \\
(1.08- \\
2.41)^{\star}\end{array}$ & $\begin{array}{l}1.15 \\
(0.75- \\
1.76)\end{array}$ \\
\hline No & $\begin{array}{l}5341 \\
(69.3)\end{array}$ & 59 & 1.1 & 1.00 & 1.00 \\
\hline \multicolumn{6}{|l|}{ Parity } \\
\hline $1-2$ & $\begin{array}{l}1945 \\
(25.2)\end{array}$ & 24 & 1.2 & 1.00 & \\
\hline
\end{tabular}

*significant at $p<0.05 ;$ na $=$ not estimated due to lack of data 


\begin{tabular}{|c|c|c|c|c|c|}
\hline Variable & $\begin{array}{l}\text { Number } \\
\text { of } \\
\text { women } \\
(\%)\end{array}$ & $\begin{array}{l}\text { Number of } \\
\text { women with } \\
\text { heart disease }\end{array}$ & $\begin{array}{l}\text { Percentage (\%) of } \\
\text { women with heart } \\
\text { disease }\end{array}$ & $\begin{array}{l}\text { Unadjusted } \\
\text { OR (95\%Cl) }\end{array}$ & $\begin{array}{l}\text { Adjusted } \\
\text { OR } \\
(95 \% \mathrm{Cl})\end{array}$ \\
\hline $3-4$ & $\begin{array}{l}1691 \\
(21.9)\end{array}$ & 31 & 1.8 & $\begin{array}{l}1.49 \\
(0.87- \\
2.55)\end{array}$ & \\
\hline$>4$ & $\begin{array}{l}2027 \\
(26.3)\end{array}$ & 33 & 1.6 & $\begin{array}{l}1.32 \\
(0.78- \\
2.25)\end{array}$ & \\
\hline No birth & $\begin{array}{l}2049 \\
(26.6)\end{array}$ & 13 & 0.6 & $\begin{array}{l}0.51 \\
(0.26- \\
1.01)\end{array}$ & \\
\hline \multicolumn{6}{|l|}{$\begin{array}{l}\text { Total lifetime } \\
\text { number of sex } \\
\text { partners }\end{array}$} \\
\hline 1 & $\begin{array}{l}3067 \\
(45.5)\end{array}$ & 36 & 1.2 & 1.00 & \\
\hline 2 & $\begin{array}{l}2121 \\
(31.4)\end{array}$ & 34 & 1.6 & $\begin{array}{l}1.37 \\
(0.86- \\
2.20)\end{array}$ & \\
\hline 3 & $\begin{array}{l}955 \\
(14.2)\end{array}$ & 17 & 1.8 & $\begin{array}{l}1.53 \\
(0.85- \\
2.73)\end{array}$ & \\
\hline$>3$ & 603 (8.9) & 11 & 1.8 & $\begin{array}{l}1.56 \\
(0.79- \\
3.09)\end{array}$ & \\
\hline \multicolumn{6}{|l|}{ Religion } \\
\hline Christianity & $\begin{array}{l}4230 \\
(54.9)\end{array}$ & 67 & 1.6 & 1.00 & 1.00 \\
\hline Islam & $\begin{array}{l}2298 \\
(29.8)\end{array}$ & 19 & 0.8 & $\begin{array}{l}0.52 \\
(0.31- \\
0.86)^{\star}\end{array}$ & $\begin{array}{l}0.99 \\
(0.53- \\
1.84)\end{array}$ \\
\hline $\begin{array}{l}\text { Traditional and } \\
\text { others }\end{array}$ & $\begin{array}{l}1184 \\
(15.3)\end{array}$ & 15 & 1.3 & $\begin{array}{l}0.80 \\
(0.45- \\
1.40)\end{array}$ & $\begin{array}{l}0.95 \\
(0.51- \\
1.74)\end{array}$ \\
\hline \multicolumn{6}{|l|}{ Maternal age } \\
\hline $15-19$ & $\begin{array}{l}1623 \\
(21.1)\end{array}$ & 7 & 0.4 & 1.00 & 1.00 \\
\hline
\end{tabular}

*significant at $p<0.05 ;$ na = not estimated due to lack of data 


\begin{tabular}{|c|c|c|c|c|c|}
\hline Variable & $\begin{array}{l}\text { Number } \\
\text { of } \\
\text { women } \\
\text { (\%) }\end{array}$ & $\begin{array}{l}\text { Number of } \\
\text { women with } \\
\text { heart disease }\end{array}$ & $\begin{array}{l}\text { Percentage }(\%) \text { of } \\
\text { women with heart } \\
\text { disease }\end{array}$ & $\begin{array}{l}\text { Unadjusted } \\
\text { OR }(95 \% \mathrm{Cl})\end{array}$ & $\begin{array}{l}\text { Adjusted } \\
\text { OR } \\
(95 \% \mathrm{Cl})\end{array}$ \\
\hline $20-24$ & $\begin{array}{l}1422 \\
(18.4)\end{array}$ & 12 & 0.8 & $\begin{array}{l}1.96(0.77- \\
5.00)\end{array}$ & $\begin{array}{l}1.72 \\
(0.62- \\
4.79)\end{array}$ \\
\hline $25-29$ & $\begin{array}{l}1488 \\
(19.3)\end{array}$ & 18 & 1.2 & $\begin{array}{l}2.83 \\
(1.18- \\
6.79)^{\star}\end{array}$ & $\begin{array}{l}2.17 \\
(0.75- \\
6.29)\end{array}$ \\
\hline $30-34$ & $\begin{array}{l}1029 \\
(13.3)\end{array}$ & 21 & 2.0 & $\begin{array}{l}4.81 \\
(2.04- \\
11.35)^{\star}\end{array}$ & $\begin{array}{l}3.49 \\
(1.18- \\
10.31)^{\star}\end{array}$ \\
\hline $35-39$ & $\begin{array}{l}942 \\
(12.2)\end{array}$ & 17 & 1.8 & $\begin{array}{l}4.24 \\
(1.75- \\
10.27)^{\star}\end{array}$ & $\begin{array}{l}2.66 \\
(0.87- \\
8.13)\end{array}$ \\
\hline $40-44$ & $613(8.0)$ & 13 & 2.1 & $\begin{array}{l}5.00 \\
(1.99- \\
12.60)^{\star}\end{array}$ & $\begin{array}{l}2.86 \\
(0.90- \\
9.09)\end{array}$ \\
\hline $45-49$ & $595(7.7)$ & 13 & 2.2 & $\begin{array}{l}5.16 \\
(2.05- \\
12.99)^{\star}\end{array}$ & $\begin{array}{l}2.66 \\
(0.83- \\
8.47)\end{array}$ \\
\hline \multicolumn{6}{|c|}{$\begin{array}{l}\text { Geographical } \\
\text { region }\end{array}$} \\
\hline Alibori & $\begin{array}{l}861 \\
(11.2)\end{array}$ & 7 & 0.8 & 1.00 & 1.00 \\
\hline Atacora & $666(8.6)$ & 8 & 1.2 & $\begin{array}{l}1.48 \\
(0.54- \\
4.11)\end{array}$ & $\begin{array}{l}1.64 \\
(0.56- \\
4.83)\end{array}$ \\
\hline Atlantic & $\begin{array}{l}808 \\
(10.5)\end{array}$ & 27 & 3.3 & $\begin{array}{l}4.22 \\
(1.83- \\
9.74)^{\star}\end{array}$ & $\begin{array}{l}3.34 \\
(1.27- \\
8.79)^{\star}\end{array}$ \\
\hline Borgou & $\begin{array}{l}858 \\
(11.1)\end{array}$ & 3 & 0.4 & $\begin{array}{l}0.43 \\
(0.11- \\
1.66)\end{array}$ & $\begin{array}{l}0.45 \\
(0.11- \\
1.75)\end{array}$ \\
\hline Collines & 716 (9.3) & 2 & 0.3 & $\begin{array}{l}0.34 \\
(0.07- \\
1.65)\end{array}$ & $\begin{array}{l}0.33 \\
(0.06- \\
1.70)\end{array}$ \\
\hline Couffo & $493(6.4)$ & 4 & 0.8 & $\begin{array}{l}1.00 \\
(0.29- \\
3.43)\end{array}$ & $\begin{array}{l}0.89 \\
(0.23- \\
3.44)\end{array}$ \\
\hline Donga & 451 (5.9) & 0 & 0.0 & na & na \\
\hline
\end{tabular}




\begin{tabular}{|c|c|c|c|c|c|}
\hline Variable & $\begin{array}{l}\text { Number } \\
\text { of } \\
\text { women } \\
\text { (\%) }\end{array}$ & $\begin{array}{l}\text { Number of } \\
\text { women with } \\
\text { heart disease }\end{array}$ & $\begin{array}{l}\text { Percentage (\%) of } \\
\text { women with heart } \\
\text { disease }\end{array}$ & $\begin{array}{l}\text { Unadjusted } \\
\text { OR }(95 \% \mathrm{Cl})\end{array}$ & $\begin{array}{l}\text { Adjusted } \\
\text { OR } \\
\text { (95\% } \mathrm{Cl})\end{array}$ \\
\hline Littoral & $661(8.6)$ & 15 & 2.3 & $\begin{array}{l}2.83 \\
(1.14- \\
6.99)^{\star}\end{array}$ & $\begin{array}{l}1.91 \\
(0.69- \\
5.28)\end{array}$ \\
\hline Mono & $398(5.2)$ & 4 & 1.0 & $\begin{array}{l}1.24 \\
(0.36- \\
4.26)\end{array}$ & $\begin{array}{l}1.19 \\
(0.31- \\
4.54)\end{array}$ \\
\hline Oueme & $616(8.0)$ & 9 & 1.5 & $\begin{array}{l}1.81 \\
(0.67- \\
4.88)\end{array}$ & $\begin{array}{l}1.33 \\
(0.45- \\
3.95)\end{array}$ \\
\hline Plateau & $465(6.0)$ & 15 & 3.2 & $\begin{array}{l}4.07 \\
(1.65- \\
10.05)^{\star}\end{array}$ & $\begin{array}{l}3.20 \\
(1.19- \\
8.57)^{\star}\end{array}$ \\
\hline Zou & 719 (9.3) & 7 & 1.0 & $\begin{array}{l}1.20 \\
(0.42- \\
3.44)\end{array}$ & $\begin{array}{l}1.08 \\
(0.34- \\
3.46)\end{array}$ \\
\hline \multicolumn{6}{|l|}{ Place of residence } \\
\hline Urban & $\begin{array}{l}3431 \\
(44.5)\end{array}$ & 44 & 1.3 & 1.00 & \\
\hline Rural & $\begin{array}{l}4281 \\
(55.5)\end{array}$ & 57 & 1.3 & $\begin{array}{l}1.04 \\
(0.70- \\
1.54)\end{array}$ & \\
\hline \multicolumn{6}{|l|}{ Marital status } \\
\hline Never married & $\begin{array}{l}1918 \\
(24.9)\end{array}$ & 12 & 0.6 & 1.00 & 1.00 \\
\hline Currently married & $\begin{array}{l}5406 \\
(70.1)\end{array}$ & 75 & 1.4 & $\begin{array}{l}2.23 \\
(1.21- \\
4.11)^{\star}\end{array}$ & $\begin{array}{l}1.03 \\
(0.45- \\
2.33)\end{array}$ \\
\hline Formerly married & $388(5.0)$ & 14 & 3.6 & $\begin{array}{l}5.95 \\
(2.73- \\
12.96)^{\star}\end{array}$ & $\begin{array}{l}1.75 \\
(0.65- \\
4.74)\end{array}$ \\
\hline \multicolumn{6}{|l|}{$\begin{array}{l}\text { Maternal } \\
\text { education }\end{array}$} \\
\hline $\begin{array}{l}\text { No formal } \\
\text { education }\end{array}$ & $\begin{array}{l}4236 \\
(54.9)\end{array}$ & 53 & 1.3 & 1.00 & \\
\hline Primary & $\begin{array}{l}1516 \\
(19.7)\end{array}$ & 22 & 1.5 & $\begin{array}{l}1.16 \\
(0.70- \\
1.92)\end{array}$ & \\
\hline
\end{tabular}




\begin{tabular}{|c|c|c|c|c|c|}
\hline Variable & $\begin{array}{l}\text { Number } \\
\text { of } \\
\text { women } \\
(\%)\end{array}$ & $\begin{array}{l}\text { Number of } \\
\text { women with } \\
\text { heart disease }\end{array}$ & $\begin{array}{l}\text { Percentage (\%) of } \\
\text { women with heart } \\
\text { disease }\end{array}$ & $\begin{array}{l}\text { Unadjusted } \\
\text { OR }(95 \% \mathrm{Cl})\end{array}$ & $\begin{array}{l}\text { Adjusted } \\
\text { OR } \\
(95 \% \mathrm{Cl})\end{array}$ \\
\hline Secondary+ & $\begin{array}{l}1960 \\
(25.4)\end{array}$ & 26 & 1.3 & $\begin{array}{l}1.06 \\
(0.66- \\
1.70)\end{array}$ & \\
\hline \multicolumn{6}{|l|}{ Wealth index } \\
\hline Poor & $\begin{array}{l}2996 \\
(3880)\end{array}$ & 39 & 1.3 & 1.00 & \\
\hline Middle & $\begin{array}{l}1359 \\
(17.6)\end{array}$ & 11 & 0.8 & $\begin{array}{l}0.62 \\
(0.32- \\
1.21)\end{array}$ & \\
\hline Rich & $\begin{array}{l}3357 \\
(43.5)\end{array}$ & 51 & 1.5 & $\begin{array}{l}1.17 \\
(0.77- \\
1.78)\end{array}$ & \\
\hline \multicolumn{6}{|c|}{$\begin{array}{l}\text { Participation in } \\
\text { labour force }\end{array}$} \\
\hline Working & $\begin{array}{l}5833 \\
(75.6)\end{array}$ & 80 & 1.4 & $\begin{array}{l}1.23(0.76- \\
2.00)\end{array}$ & \\
\hline Not working & $\begin{array}{l}1879 \\
(24.4)\end{array}$ & 21 & 1.1 & 1.00 & \\
\hline \multicolumn{6}{|c|}{$\begin{array}{l}\text { Covered by health } \\
\text { insurance }\end{array}$} \\
\hline Not covered & $\begin{array}{l}7628 \\
(98.9)\end{array}$ & 99 & 1.3 & 1.00 & \\
\hline Covered & $84(1.1)$ & 2 & 2.4 & $\begin{array}{l}1.85 \\
(0.45- \\
7.65)\end{array}$ & \\
\hline
\end{tabular}

The prevalence of lung disease was approximately $1.5 \%$ (95\% Cl: $1.2 \%-1.9 \%)$. Results from Table 2 showed that approximately $9.6 \%$ of those with diabetes had lung disease, in contrast to $1.6 \%$ of those who do not have diabetes, but had lung disease. Women with diabetes were 4.55 times more likely to have lung diseases, when compared with those who do not have diabetes ( $\mathrm{AOR}=4.55 ; 95 \% \mathrm{Cl}$ : $2.06-$ 10.06). In addition, the geographical region was significantly associated with lung disease do not have hypertension and diabetes (See Table 2 for the details). 
Table 2

Association between diabetes and correlates of lung disease among women aged 15-49 years in Benin Variable $\begin{array}{ll}\begin{array}{l}\text { Number } \\ \text { of }\end{array} & \begin{array}{l}\text { Number of } \\ \text { women with }\end{array} \\ \text { women } & \text { lung disease }\end{array}$ Percentage (\%) of women with lung

(\%) disease

Adjusted $\begin{array}{ll}\text { Unadjusted } & \text { Adj } \\ \text { OR }(95 \% \mathrm{Cl}) & \text { OR }\end{array}$ $(95 \% \mathrm{Cl})$

\section{Hypertension}

\begin{tabular}{lllll} 
Yes & $751(9.7)$ & 19 & 2.5 & $\begin{array}{l}1.63 \\
(0.99- \\
2.67)\end{array}$ \\
& & & & 1.00 \\
\hline No & 6961 & 109 & 1.6 & \\
\hline
\end{tabular}

\section{Diabetes}

Yes

$83(1.1) \quad 8$

9.6

6.67

(3.15-

4.55

14.14)*

(2.06-

1.00

No

7629

(98.9)

120

1.6

1.00

1.00

\section{Usage of}

cigarettes or

tobacco products

$\begin{array}{lllll}\text { Use } & 216(2.8) & 6 & 2.8 & 1.00 \\ \text { Not use } & 7496 & 122 & 1.6 & 0.58 \\ & (97.2) & & & (0.25- \\ & & & & 1.33)\end{array}$

\section{Ever used any}

method to delay

pregnancy

\begin{tabular}{cccccc} 
Yes & 2371 & 52 & 2.2 & 1.55 & 1.30 \\
& $(30.7)$ & & & $\begin{array}{l}(1.09- \\
2.22)^{*}\end{array}$ & $\begin{array}{l}(0.88- \\
1.94)\end{array}$ \\
\hline No & & & & 1.00 & 1.00 \\
\hline
\end{tabular}

\section{Parity}

$1-2$

1945

(25.2)

3-4

1691

(21.9)

29

1.5

1.00

33

2.0

1.32

(0.80-

2.17)

*significant at $p<0.05$ 


\begin{tabular}{|c|c|c|c|c|c|}
\hline Variable & $\begin{array}{l}\text { Number } \\
\text { of } \\
\text { women } \\
(\%)\end{array}$ & $\begin{array}{l}\text { Number of } \\
\text { women with } \\
\text { lung disease }\end{array}$ & $\begin{array}{l}\text { Percentage (\%) of } \\
\text { women with lung } \\
\text { disease }\end{array}$ & $\begin{array}{l}\text { Unadjusted } \\
\text { OR (95\%Cl) }\end{array}$ & $\begin{array}{l}\text { Adjusted } \\
\text { OR } \\
(95 \% \mathrm{Cl})\end{array}$ \\
\hline$>4$ & $\begin{array}{l}2027 \\
(26.3)\end{array}$ & 30 & 1.5 & $\begin{array}{l}0.99 \\
(0.59- \\
1.66)\end{array}$ & \\
\hline No birth & $\begin{array}{l}2049 \\
(26.6)\end{array}$ & 36 & 1.8 & $\begin{array}{l}1.18 \\
(0.72- \\
1.93)\end{array}$ & \\
\hline \multicolumn{6}{|l|}{$\begin{array}{l}\text { Total lifetime } \\
\text { number of sex } \\
\text { partners }\end{array}$} \\
\hline 1 & $\begin{array}{l}3067 \\
(45.5)\end{array}$ & 45 & 1.5 & 1.00 & 1.00 \\
\hline 2 & $\begin{array}{l}2121 \\
(31.4)\end{array}$ & 42 & 2.0 & $\begin{array}{l}1.36 \\
(0.89- \\
2.07)\end{array}$ & $\begin{array}{l}1.17 \\
(0.76- \\
1.82)\end{array}$ \\
\hline 3 & $\begin{array}{l}955 \\
(14.2)\end{array}$ & 11 & 1.2 & $\begin{array}{l}0.78 \\
(0.40- \\
1.52)\end{array}$ & $\begin{array}{l}0.56 \\
(0.28- \\
1.12)\end{array}$ \\
\hline$>3$ & 603 (8.9) & 16 & 2.7 & $\begin{array}{l}1.83 \\
(1.03- \\
3.26)^{*}\end{array}$ & $\begin{array}{l}1.26 \\
(0.67- \\
2.35)\end{array}$ \\
\hline \multicolumn{6}{|l|}{ Religion } \\
\hline Christianity & $\begin{array}{l}4230 \\
(54.9)\end{array}$ & 83 & 2.0 & 1.00 & 1.00 \\
\hline Islam & $\begin{array}{l}2298 \\
(29.8)\end{array}$ & 29 & 1.3 & $\begin{array}{l}0.64 \\
(0.42- \\
0.98)^{\star}\end{array}$ & $\begin{array}{l}1.49 \\
(0.87- \\
2.56)\end{array}$ \\
\hline $\begin{array}{l}\text { Traditional and } \\
\text { others }\end{array}$ & $\begin{array}{l}1184 \\
(15.3)\end{array}$ & 16 & 1.4 & $\begin{array}{l}0.68 \\
(0.40- \\
1.17)\end{array}$ & $\begin{array}{l}1.07 \\
(0.58- \\
1.96)\end{array}$ \\
\hline \multicolumn{6}{|l|}{ Maternal age } \\
\hline $15-19$ & $\begin{array}{l}1623 \\
(21.1)\end{array}$ & 20 & 1.2 & 1.00 & \\
\hline $20-24$ & $\begin{array}{l}1422 \\
(18.4)\end{array}$ & 24 & 1.7 & $\begin{array}{l}1.38 \\
(0.76- \\
2.50)\end{array}$ & \\
\hline
\end{tabular}

*significant at $p<0.05$ 


\begin{tabular}{|c|c|c|c|c|c|}
\hline Variable & $\begin{array}{l}\text { Number } \\
\text { of } \\
\text { women } \\
\text { (\%) }\end{array}$ & $\begin{array}{l}\text { Number of } \\
\text { women with } \\
\text { lung disease }\end{array}$ & $\begin{array}{l}\text { Percentage (\%) of } \\
\text { women with lung } \\
\text { disease }\end{array}$ & $\begin{array}{l}\text { Unadjusted } \\
\text { OR }(95 \% \mathrm{Cl})\end{array}$ & $\begin{array}{l}\text { Adjusted } \\
\text { OR } \\
(95 \% \mathrm{Cl})\end{array}$ \\
\hline $25-29$ & $\begin{array}{l}1488 \\
(19.3)\end{array}$ & 22 & 1.5 & $\begin{array}{l}1.20 \\
(0.65- \\
2.21)\end{array}$ & \\
\hline $30-34$ & $\begin{array}{l}1029 \\
(13.3)\end{array}$ & 22 & 2.1 & $\begin{array}{l}1.75 \\
(0.95- \\
3.22)\end{array}$ & \\
\hline $35-39$ & $\begin{array}{l}942 \\
(12.2)\end{array}$ & 19 & 2.0 & $\begin{array}{l}1.65 \\
(0.88- \\
3.11)\end{array}$ & \\
\hline $40-44$ & $613(8.0)$ & 12 & 2.0 & $\begin{array}{l}1.60 \\
(0.78- \\
3.29)\end{array}$ & \\
\hline $45-49$ & 595 (7.7) & 9 & 1.5 & $\begin{array}{l}1.23 \\
(0.56- \\
2.72)\end{array}$ & \\
\hline \multicolumn{6}{|c|}{$\begin{array}{l}\text { Geographical } \\
\text { region }\end{array}$} \\
\hline Alibori & $\begin{array}{l}861 \\
(11.2)\end{array}$ & 3 & 0.4 & 1.00 & 1.00 \\
\hline Atacora & 666 (8.6) & 8 & 1.2 & $\begin{array}{l}3.48 \\
(0.92- \\
13.16)\end{array}$ & $\begin{array}{l}3.50 \\
(0.87- \\
14.12)\end{array}$ \\
\hline Atlantic & $\begin{array}{l}808 \\
(10.5)\end{array}$ & 26 & 3.2 & $\begin{array}{l}9.51 \\
(2.87- \\
31.54)^{\star}\end{array}$ & $\begin{array}{l}10.98 \\
(3.02- \\
39.98)^{\star}\end{array}$ \\
\hline Borgou & $\begin{array}{l}858 \\
(11.1)\end{array}$ & 10 & 1.2 & $\begin{array}{l}3.37 \\
(0.92- \\
12.30)\end{array}$ & $\begin{array}{l}3.37 \\
(0.92- \\
12.37)\end{array}$ \\
\hline Collines & $716(9.3)$ & 27 & 3.8 & $\begin{array}{l}11.21 \\
(3.39- \\
37.10)^{\star}\end{array}$ & $\begin{array}{l}9.74 \\
(2.70- \\
35.07)^{\star}\end{array}$ \\
\hline Couffo & $493(6.4)$ & 7 & 1.4 & $\begin{array}{l}4.12(1.06- \\
16.00)^{\star}\end{array}$ & $\begin{array}{l}6.64 \\
(1.56- \\
28.23)^{\star}\end{array}$ \\
\hline Donga & 451 (5.9) & 2 & 0.4 & $\begin{array}{l}1.27 \\
(0.21- \\
7.65)\end{array}$ & $\begin{array}{l}1.36 \\
(0.22- \\
8.22)\end{array}$ \\
\hline
\end{tabular}




\begin{tabular}{|c|c|c|c|c|c|}
\hline Variable & $\begin{array}{l}\text { Number } \\
\text { of } \\
\text { women } \\
(\%)\end{array}$ & $\begin{array}{l}\text { Number of } \\
\text { women with } \\
\text { lung disease }\end{array}$ & $\begin{array}{l}\text { Percentage (\%) of } \\
\text { women with lung } \\
\text { disease }\end{array}$ & $\begin{array}{l}\text { Unadjusted } \\
\text { OR (95\%CI) }\end{array}$ & $\begin{array}{l}\text { Adjusted } \\
\text { OR } \\
(95 \% \mathrm{Cl})\end{array}$ \\
\hline Littoral & $661(8.6)$ & 20 & 3.0 & $\begin{array}{l}8.92 \\
(2.64- \\
30.16)^{*}\end{array}$ & $\begin{array}{l}7.84 \\
(2.13- \\
28.91)^{\star}\end{array}$ \\
\hline Mono & $398(5.2)$ & 1 & 0.3 & $\begin{array}{l}0.72 \\
(0.07- \\
6.95)\end{array}$ & $\begin{array}{l}0.96 \\
(0.09- \\
9.76)\end{array}$ \\
\hline Oueme & 616 (8.0) & 9 & 1.5 & $\begin{array}{l}4.24 \\
(1.14- \\
15.73)^{\star}\end{array}$ & $\begin{array}{l}4.90 \\
(1.22- \\
19.68)^{\star}\end{array}$ \\
\hline Plateau & $465(6.0)$ & 6 & 1.3 & $\begin{array}{l}3.74 \\
(0.93- \\
15.02)\end{array}$ & $\begin{array}{l}4.25 \\
(0.97- \\
18.56)\end{array}$ \\
\hline Zou & 719 (9.3) & 9 & 1.3 & $\begin{array}{l}3.63 \\
(0.98- \\
13.44)\end{array}$ & $\begin{array}{l}4.90 \\
(1.22- \\
19.72)^{\star}\end{array}$ \\
\hline \multicolumn{6}{|l|}{ Place of residence } \\
\hline Urban & $\begin{array}{l}3431 \\
(44.5)\end{array}$ & 62 & 1.8 & 1.00 & \\
\hline Rural & $\begin{array}{l}4281 \\
(55.5)\end{array}$ & 66 & 1.5 & $\begin{array}{l}0.85 \\
(0.60- \\
1.21)\end{array}$ & \\
\hline \multicolumn{6}{|l|}{ Marital status } \\
\hline Never married & $\begin{array}{l}1918 \\
(24.9)\end{array}$ & 36 & 1.9 & 1.00 & 1.00 \\
\hline Currently married & $\begin{array}{l}5406 \\
(70.1)\end{array}$ & 78 & 1.4 & $\begin{array}{l}0.77 \\
(0.51- \\
1.14)\end{array}$ & $\begin{array}{l}0.80 \\
(0.48- \\
1.34)\end{array}$ \\
\hline Formerly married & $388(5.0)$ & 14 & 3.6 & $\begin{array}{l}1.96 \\
(1.05- \\
3.66)^{\star}\end{array}$ & $\begin{array}{l}1.38 \\
(0.65- \\
2.90)\end{array}$ \\
\hline \multicolumn{6}{|l|}{$\begin{array}{l}\text { Maternal } \\
\text { education }\end{array}$} \\
\hline $\begin{array}{l}\text { No formal } \\
\text { education }\end{array}$ & $\begin{array}{l}4236 \\
(54.9)\end{array}$ & 54 & 1.3 & 1.00 & 1.00 \\
\hline Primary & $\begin{array}{l}1516 \\
(19.7)\end{array}$ & 25 & 1.7 & $\begin{array}{l}1.30 \\
(0.81- \\
2.09)\end{array}$ & $\begin{array}{l}0.93 \\
(0.55- \\
1.59)\end{array}$ \\
\hline
\end{tabular}




\begin{tabular}{|c|c|c|c|c|c|}
\hline Variable & $\begin{array}{l}\text { Number } \\
\text { of } \\
\text { women } \\
(\%)\end{array}$ & $\begin{array}{l}\text { Number of } \\
\text { women with } \\
\text { lung disease }\end{array}$ & $\begin{array}{l}\text { Percentage (\%) of } \\
\text { women with lung } \\
\text { disease }\end{array}$ & $\begin{array}{l}\text { Unadjusted } \\
\text { OR }(95 \% \mathrm{Cl})\end{array}$ & $\begin{array}{l}\text { Adjusted } \\
\text { OR } \\
(95 \% \mathrm{Cl})\end{array}$ \\
\hline Secondary+ & $\begin{array}{l}1960 \\
(25.4)\end{array}$ & 49 & 2.5 & $\begin{array}{l}1.99 \\
(1.34- \\
2.93)^{\star}\end{array}$ & $\begin{array}{l}1.49 \\
(0.93- \\
2.40)\end{array}$ \\
\hline \multicolumn{6}{|l|}{ Wealth index } \\
\hline Poor & $\begin{array}{l}2996 \\
(3880)\end{array}$ & 43 & 1.4 & 1.00 & \\
\hline Middle & $\begin{array}{l}1359 \\
(17.6)\end{array}$ & 15 & 1.1 & $\begin{array}{l}0.77 \\
(0.42- \\
1.38)\end{array}$ & \\
\hline Rich & $\begin{array}{l}3357 \\
(43.5)\end{array}$ & 70 & 2.1 & $\begin{array}{l}1.46 \\
(0.99- \\
2.14)\end{array}$ & \\
\hline \multicolumn{6}{|c|}{$\begin{array}{l}\text { Participation in } \\
\text { labour force }\end{array}$} \\
\hline Working & $\begin{array}{l}5833 \\
(75.6)\end{array}$ & 104 & 1.8 & $\begin{array}{l}1.40 \\
(0.90- \\
2.19)\end{array}$ & \\
\hline Not working & $\begin{array}{l}1879 \\
(24.4)\end{array}$ & 24 & 1.3 & 1.00 & \\
\hline \multicolumn{6}{|c|}{$\begin{array}{l}\text { Covered by health } \\
\text { insurance }\end{array}$} \\
\hline Not covered & $\begin{array}{l}7628 \\
(98.9)\end{array}$ & 127 & 1.7 & 1.00 & \\
\hline Covered & $84(1.1)$ & 1 & 1.2 & $\begin{array}{l}0.71 \\
(0.10- \\
5.15)\end{array}$ & \\
\hline
\end{tabular}

\section{Discussion}

This study is the first of its kind in the provision of a nationwide report of the prevalence of heart and lung diseases among women of reproductive age in the Republic of Benin, West Africa. Some studies from other SSA countries have reported findings similar to the one reported here. However, no report has been made on the status of heart and lung diseases and their relationship with hypertension and diabetes among reproductive-age women in Benin Republic.

The prevalence of lung and heart diseases as observed in this study were approximately $2 \%$ each. This is similar to the $2.4 \%$ reported in Yaounde, Cameroon (30), but lower than 20.2\% reported in urban and rural 
Uganda (31) and 17.8\% reported in Abeshge District of Ethiopia (32). Reports by several researchers show that the prevalence of chronic obstructive pulmonary disease, which is the third leading cause of mortality caused by non-communicable diseases globally (10), in SSA ranges from $4.1 \%-22.2 \%$ (33). The difference observed in this study may be interpreted to be due to the fact that this study utilizes a national data and in this case, only the known cases of lung and heart diseases are reported as the interviewers did not screen the participants to clearly determine those that have lung and hearts diseases, but depended on the medical history supplied to them by the participants. Therefore, there may be some study participants who may have lung or heart disease but because she has not been diagnosed, would have no clue that she has the disease.

This study shows that $4.7 \%$ and $8.4 \%$ of women, who had hypertension and diabetes respectively, also were with known heart diseases, in comparison to $1.0 \%$ and $1.2 \%$ of those who have heart disease but do not have hypertension and diabetes respectively. Our study observed an association between hypertension and diabetes, and heart diseases. Hypertension and diabetes were risk factors for heart disease. This association is very much in agreement with several studies findings across SSA countries, which have also come up with reports that hypertension or/and diabetes are risk factors of CVDs; such as Nigeria (34), Cameroon (35, 36), Benin (37), and 2010 Global Burden of Diseases report on CVDs in SSA (18). Mandi et al, also reported that hypertension was the most prevalent risk factor of CVD in rural Burkina Faso (38).

More so, a four-country SSA cross-sectional study reported that $50.0 \%$ of the people with hypertension that participated in the study were unaware of being hypertensive (39) This implies that the menace of hypertension may be much more that is currently known among SSA population, as some studies have also suggested (40-42). This therefore, suggests that a large proportion of the region's hypertension cases remain undiagnosed, untreated, or inadequately treated, hence may be the highest contributor for morbidity and mortality caused by complications of CVD (42-44). Over the past decades, in SSA, the attention of governments and funding agencies have been directed to the fight against communicable diseases such as HIV/AIDS, Tuberculosis, Malaria, with NCDs being neglected or relegated to the background $(3,4)$. The burden of hypertension is such a public health concern as reports have it that it is the single leading cause of death and hospitalization globally $(41,45-47)$. The current trends in globalization may have contributed to the rise in the cases of non-communicable diseases in many low and middle-income countries such as SSA countries as lifestyles and dietary habits' changes have occurred among people in low and middle-income economies (19).

Our study also found an association between diabetes and lung diseases among the studied participant. Approximately $9.6 \%$ of those with known lung disease also have diabetes compared with $1.6 \%$ who had lung diseases without elevated blood glucose. Several other reports have shown that diabetes is a comorbidity with lung diseases (48-54). The relationship between lung diseases and diabetes has been extensively studied, though the mechanism with which this association exist has not been well understood. Explanations have been offered on the possible mechanisms; that hyperglycemic effects on the physiologic status of the lungs, inflammatory responses or the lungs susceptibility to infections may 
be a significant contributor to this association $(55,56)$, another possible mechanism is offered by Zheng et al, which attributed the association to a sustained diabetes level resulting in oxidative stress (OS) thereby causing tissue damage (57). Aside from these possibilities, lifestyles such as tobacco use, sedentary life, physical inactivity, air pollution as well as age have been implicated as possible risk factors for both heart and lung diseases $(37,51,58-60)$.

This study observed that geographical departments and age of the participants are determining factors for heart and lung diseases. Benin Republic has twelve departments with three of them being essentially urban; Oueme, Littoral and Atlantic, another three are essentially rural; Atakora, Borgou and Zou, while the rest are essentially semi-urban $(61,62)$. We observed that participants from the rural departments were found to be less likely to have heart and lung diseases compared with participants from the essentially urban departments. This finding is in corroboration with the report from South Africa; it was reported that the most developed areas in the study recorded higher heart and lungs diseases (63). Another study has reported that the burden of heart-related diseases was in the urban areas and the densely populated part of the city (60). The study also revealed that the majority of the CVDs were in the elite and middle-class neighborhoods.

Therefore, CVD was predominantly high in rich environment, while generally low in the middle-income and more rural/urban sprawl neighborhoods (60). Two South African studies also are in agreement that heart diseases are higher in urban areas than in rural areas $(64,65)$. The high prevalence observed among participants from urban departments compared with those from the essentially rural departments can be explained in the view of industrialization and urbanization (urban cities come with much day-to-day hustles to catch up with day's activities, stress from traffics, and vehicular as well as industrial pollutions). People in urban cities are also prone to poor dietary lifestyle as majority often depend on junk foods, sedentary lifestyle or physical inactivity due to office work, tobacco use, compared with rural dwellers. Our study revealed that women from the mostly urban departments were more likely to have heart and lung diseases compared with those in the rural departments.

\section{Strengths and limitations}

The major strength of this study is the use of nationally representative data and the findings are generalizable for the women of reproductive age in Benin, West Africa. However, only an association of the factors and not causation can be inferred due to the cross-sectional nature of the data. Also, we were unable to explore other contributory risk factors of heart and lung diseases such as overweight/obesity, salt intake, psychosocial stress, and other endogenous factors.

\section{Conclusion}

We found that hypertension was associated with heart disease among women aged 15-49 years in Benin. Health policymakers and government need to focus on widespread prevention and control interventions of heart and lung diseases through improved screening for risk factors and early detection 
of CVDs especially among high-risk women. The government should create nationwide awareness and educate people about CVDs and the fatality rate.

\section{Declarations}

Ethics approval and consent to participate

Not Applicable.

Consent for publication

Not Applicable.

\section{Availability of data and materials}

Data for this study were sourced from Demographic and Health surveys (DHS) and available here: https://www.dhsprogram.com/data/available-datasets.cfm

\section{Competing interests}

The authors declare that they have no competing interests.

\section{Funding}

The authors have no support or funding to report.

\section{Authors' contributions}

ME conceptualised the study, prepared the study design, analyzed the data and wrote the results. $\mathrm{CIN}$ and $A B$ reviewed literature and discussed the findings. $M E, C I N$ and $A B$ made substantial inputs in the manuscripts and critically reviewed the manuscript for its intellectual content. All authors read and approved the manuscript.

\section{Acknowledgment}

The authors thank the MEASURE DHS project for their support and for free access to the original data.

\section{Abbreviations}

AIDS Acquired immunodeficiency syndrome 

AOR Adjusted odds ratio
BDHS Benin Demographic and Health Survey
$\mathrm{Cl} \quad$ Confidence interval
COPD Chronic obstructive pulmonary disease

CVDs Cardiovascular diseases

DALYs Disability-Adjusted Life-Years

EAs Enumeration areas

GBDs Global Burden of Diseases

HIV Human immunodeficiency virus

ICF Inner City Fund

LGA Local Government Area

NCDs Non-communicable diseases

NPC National Population Commission

PCA Principal Component Analysis

SDGs Sustainable Development Goals

SSA Sub-Saharan Africa

UNFPA United Nations Population Fund

UNICEF United Nations Children's Fund

USAID United States Agency for International Development

\section{References}

1. Clark H. NCDs: a challenge to sustainable human development. The Lancet. 2013;381(9866):510-1.

2. World Health Organization (WHO). Global action plan for the prevention and control of noncommunicable diseases 2013-2020. World Health Organization. 2020. 
3. Gouda HN, Charlson F, Sorsdahl K, Ahmadzada S, Ferrari AJ, Erskine H, et al. Articles Burden of noncommunicable diseases in sub-Saharan Africa, 1990-2017 : results from the Global Burden of Disease Study. The Lancet Global Health. 2017;7(10):e1375-87.

4. GBD 2017 Causes of Death Collaborators. Global, regional, and national age-sex-specific mortality for 282 causes of death in 195 countries and territories, 1980-2017: a systematic analysis for the Global Burden of Disease Study 2017. Lancet. 2018;392:1736-88.

5. GBD 2017 DALYs and. Collaborators HALE. Global, regional, and national disability-adjusted lifeyears (DALYs) for 359 diseases and injuries and healthy life expectancy (HALE) for 195 countries and territories, 1990-2017 : a systematic analysis for the Global Burden of Disease Study 201. Lancet. 2018;392:1859-922.

6. Kontis V, Mathers CD, Bonita R, Stevens GA, Rehm J, Shield KD, et al. Regional contributions of six preventable risk factors to achieving the $25 \times 25$ non-communicable disease mortality reduction target: a modelling study. Lancet Global Health. 2015;3:e746-57.

7. Ng M, Freeman MK, Fleming TD, Robinson M, Dwyer-lindgren L, Thomson B, et al. Smoking Prevalence and Cigarette Consumption in 187 Countries, 1980-2012. Journal of Amerian Medical Association. 2014;311(2):183-92.

8. Schwela D. Review of Urban Air Quality in Sub-Saharan Africa Region - Air Quality profile of SSA countries.

9. Global Burden of Disease Study. 2013 Collaborators. Global, regional, and national incidence, prevalence, and years lived with disability for 301 acute and chronic diseases and injuries in 188 countries, 1990-2013 : a systematic analysis for the Global Burden of Disease Study 2013. The Lancet. 2015;6736(15):1-58.

10. G B D 2013 Mortality and Causes of Death Collaborators. Global, regional, and national age - sex specifi c all-cause and cause-specifi c mortality for 240 causes of death, 1990-2013 : a systematic analysis for the Global Burden of Disease Study 2013. The Lancet. 2015;385(9963):117-71.

11. United Nations. Transforming our world: the 2030 Agenda for Sustainable Development. United Nations Sustainable knowledge platform. Sustainable Development Goals. 2015;1-40a.

12. Houehanou YC, Mizéhoun-adissoda $C$, Amidou $S$, Désormais I, Houénassi $M$, Preux $P$, et al. Feasibility of a cardiovascular cohort in a Sub-Saharan Africa community: preliminary report of the pilot project TAHES (Tanvè Health Study) in Benin. Global Health Action. 2017;10(1):1-8.

13. Dalal S, Beunza JJ, Volmink J, Adebamowo C, Bajunirwe F, Njelekela M, et al. Non-communicable diseases in sub-Saharan Africa: what we know now. International Journal of Epidemiology. 2011; (April):885-901.

14. Hall V, Thomsen RW, Henriksen O, Lohse N. Diabetes in Sub Saharan Africa 1999-2011:

Epidemiology and public health implications. a systematic review. BMC Public Health. 2011;11:56475.

15. NCD Risk Factor Collaboration (NCD-RisC) - Africa Working Group. Trends in obesity and diabetes across Africa from 1980 to 2014: an analysis of pooled population-based studies. International 
Journal of Epidemiology. 2017;(June):1421-32.

16. Meghji J, Nadeau G, Davis KJ, Wang D, Nyirenda MJ, Gordon SB, et al. Non-communicable lung disease in sub Saharan Africa: a community-based cross-sectional study of adults in urban Malawi. 2016. 1-53 p.

17. Mensah GA, Roth GA, Sampson UKA, Moran AE, Feigin VL, Forouzanfar MH, et al. Mortality from cardiovascular diseases in sub-Saharan Africa, 1990-2013: a systematic analysis of data from the Global Burden of Disease Study 2013. Cardiovascular Journal Africa. 2015;26(2):6-10.

18. Moran A, Forouzanfar M, Sampson U, Chugh S, Feigin V, Mensah G. The Epidemiology of Cardiovascular Diseases in Sub-Saharan Africa: The Global Burden of Diseases, Injuries and Risk Factors 2010 Study. Prog Cardiovasc Dis. 2013;56(3):234-9.

19. WHO. Report on the status of major health risk factors for noncommunicable diseases: WHO African Region. 2015. 2015.

20. Izutsu T, Tsutsumi A, Minas H, Thornicroft G, Patel V, Ito A. Mental health and wellbeing in the Sustainable Development Goals. The Lancet Psychiatry. 2015;2(12):1052-4.

21. Nyaaba GN, Stronks K, Aikins A, Kengne AP, Agyemang C. Tracing Africa 's progress towards implementing the Non-Communicable Diseases Global action plan 2013-2020: a synthesis of WHO country profile reports. BMC Public Health. 2017;17:297-309.

22. Peck R, Mghamba J, Vanobberghen F, Kavishe B, Rugarabamu V, Smeeth L, et al. Preparedness of Tanzanian health facilities for outpatient primary care of hypertension and diabetes: a crosssectional. The Lancet Global Health. 2014;2(5):e285-92.

23. Saraceno B, Ommeren M, Van, Batniji R, Cohen A, Gureje O, Mahoney J, et al. Barriers to improvement of mental health services in low-income and middle-income countries. Lancet. 2007;370:1164-74.

24. Corsi DJ, Neuman M, Finlay JE, Subramanian S. Demographic and health surveys: a profile. International Journal of Epidemiology. 2012 Dec 1;41(6):1602-13.

25. Worldbank. Population, total - Benin I Data [Internet]. [cited 2020 Mar 28]. Available from: https://data.worldbank.org/indicator/SP.POP.TOTL?locations=BJ.

26. Republic of Benin - Country Profile. Benin - Country Profile - Nations Online Project [Internet]. [cited 2019 Dec 13]. Available from: http://www.nationsonline.org/oneworld/benin.htm.

27. Rutstein SO, Staveteig S. Making the Demographic and Health Surveys Wealth Index Comparable. 2014; DHS Methodological Reports No. 9. Rockville, Maryland, USA: ICF International.

28. Hruschka DJ, Gerkey D, Hadley C. Estimating the absolute wealth of households. Bulletin of the World Health Organization. 2015 Jul 1;93(7):483-90.

29. Midi H, Sarkar SK, Rana S. Collinearity diagnostics of binary logistic regression model. Journal of Interdisciplinary Mathematics. 2010 Jun;13(3):253-67.

30. Walter E, Yone P, Kengne AP, Balkissou AD, Gaelle C, Fotso M, et al. Prevalence of obstructive lung disease in an African country using definitions from different international guidelines: a community based cross - sectional survey. BMC Research Notes. 2016;9:124. 
31. Siddharthan T, Grigsby M, Morgan B, Kalyesubula R, Wise RA, Kirenga B, et al. Prevalence of chronic respiratory disease in urban and rural Uganda. Bulletin of World Health Organization. 2019;97(March):318-27.

32. Woldeamanuel GG, Mingude AB, Geta TG. Prevalence of chronic obstructive pulmonary disease (COPD) and its associated factors among adults in Abeshge District, Ethiopia : a cross sectional study. BMC Pulm Med. 2019;19:181.

33. Finney LJ, Feary JR, Gordon SB, Mortimer K. Chronic obstructive pulmonary disease in sub-Saharan Africa: a systematic review. Int J Tuberc Lung Dis. 2013;17(5):583-9.

34. Isara1 AR, Okundia PO. The burden of hypertension and diabetes mellitus in rural communities in southern Nigeria. Pan African Medical Journal. 2015;20::103. doi:10.11604/pamj.2015.20.103.5619.

35. Princewel F, Cumber SN, Kimbi JA, Nkfusai CN, Keka El, Viyoff VZ, et al. Prevalence and risk factors associated with hypertension among adults in a rural setting: the case of Ombe, Cameroon. Pan African Medical Journal. 2019;34:147. doi:10.11604/pamj.2019.34.147.17518.

36. Kingue S, Ngoe CN, Menanga AP, Jingi AM, Noubiap JJ, Fesuh B, et al. Prevalence and Risk Factors of Hypertension in Urban Areas of Cameroon: A Nationwide Population-Based Cross-Sectional Study. The Journal of Clinical Hypertension. 2015;17(10):819-24.

37. Amoussou-Guenou D, Wanvoegbe A, Hermans M, Agbodande A, Boko M, Fandi AA-G, et al. Prevalence and Risk Factors of Diabetes Mellitus in the Adult Population of Porto-Novo (Benin). Journal of Diabetes Millitus. 2015;5(3):135-40.

38. Mandi DG, Bamouni J, Naïbé DT, Yaméogo RA, Kaboré E, Kambiré Y, et al. Epidemiology and longterm prognosis of atrial fibrillation in rural African patients. The Egyptian Heart Journal. 2019;71(6):1-7.

39. Guwatudde D, Nankya-mutyoba J, Kalyesubula R, Laurence C, Adebamowo C, Ajayi I, et al. The burden of hypertension in sub-Saharan Africa: a four-country cross sectional study The burden of hypertension in sub-Saharan Africa : a four-country cross sectional study. BMC Public Health. 2015;15:1211-8.

40. Choukem SP, Kengne AP, Dehayem YM, Simo NL, Mbanya JC. Hypertension in people with diabetes in sub-Saharan Africa: Revealing the hidden face of the iceberg. Diabetes Res Clin Pract. 2007;77:293-9.

41. Ataklte F, Erqou S, Kaptoge S, Taye B, Echouffo-tcheugui JB, Kengne AP. Burden of Undiagnosed Hypertension in Sub-Saharan Africa A Systematic Review and Meta-Analysis. Hypertension. 2015;65:00-0.

42. Addo J, Smeeth L, Leon DA. Hypertension In Sub-Saharan Africa A Systematic Review. Hypertension. 2007;50:1012-9.

43. Kengne AP, Ntyintyane LM, Mayosi BM. A systematic overview of prospective cohort studies of cardiovascular disease in sub-Saharan Africa. Cardiovascular Journal of Africa. 2012;23(2):103-12.

44. Ibrahim MM, Damasceno A. Hypertension in developing countries. The Lancet. 2012;380:611-9.

45. Poulter NR, Prabhakaran D, Caulfield M. Hypertension. Lancet. 2015;386:801-12. 
46. Bromfield S, Muntner P. High Blood Pressure: The Leading Global Burden of Disease Risk Factor and the Need for Worldwide Prevention Programs. Current Hypertension Report. 2013;15(3):134-6. doi:10.1007/s11906-013-0340-9.

47. Lim SS, Vos T, Flaxman AD, Danaei G, Shibuya K, Adair-rohani H, et al. A comparative risk assessment of burden of disease and injury attributable to 67 risk factors and risk factor clusters in 21 regions, 1990-2010 : a systematic analysis for the Global Burden of Disease Study 2010. Lancet. 2012;380:2224-60.

48. Chatila WM, Thomashow BM, Minai OA, Criner GJ, Make BJ. Comorbidities in Chronic Obstructive Pulmonary Disease. Proceedings of the American Thoracic Society. 2008;5:549-55.

49. Rana JS, Mittleman MA, Sheikh J, Hu FB, Manson JE, Colditz GA, et al. Chronic Obstructive Pulmonary Disease, Asthma, and Risk of Type 2 Diabetes in. Diabetes Care. 2004;27(10):2478-84.

50. Yang JK, Feng Y, Yuan MY, Yuan SY, Fu HJ, Wu BY, et al. Plasma glucose levels and diabetes are independent predictors for mortality and morbidity in patients with SARS. Diabet Med. 2006;23:6238.

51. Hak E, Rovers MM, Kuyvenhoven MM, Schellevis FG, Verheij TJM. Incidence of GP-diagnosed respiratory tract infections according to age, gender and high-risk co-morbidity : the Second Dutch National Survey of General Practice. Family Practice. 2006;(February):291-4.

52. El-azeem IAA, Hamdy G, Amin M, Rashad A. Pulmonary function changes in diabetic lung. Egyptian Journal of Chest Diseases Tuberculosis. 2013;62(3):513-7.

53. Walter RE, Beiser A, Givelber RJ, O’Connor GTO, Gottlieb DJ. Association between Glycemic State and Lung Function. The Framingham Heart Study. American Journal of Respiratoryand Critical Care Medicine. 2003;167:911-6.

54. Pitocco D, Fuso L, Conte EG, Zaccardi F, Condoluci C, Scavone G, et al. The Diabetic Lung - A New Target Organ? The Review of Diabetes Studies. 2012;9(1):23-35.

55. Gläser S, Kruger S, Merkel M, Bramlage P, Herth FJF. Chronic Obstructive Pulmonary Disease and Diabetes Mellitus: A Systematic Review of the Literature. Respiration. 2015;89:253-64.

56. Baker EH, Archer JRH, Srivastava SA. Hyperglycemia. Lung Infection, and Inflammation. Clinical Pulmonary Medicine. 2009;16(5):258-64.

57. Zheng H, Wu J, Jin Z, Yan L. Potential Biochemical Mechanisms of Lung Injury in Diabetes. Aging Disease. 2017;8(1):7-16.

58. Noubiap JJ, Nansseu JR, Endomba FT, Ngouo A, Nkeck JR, Nyaga UF, et al. Active smoking among people with diabetes mellitus or hypertension in Africa: a systematic review and meta-analysis. Nature Scientific Reports. 2019;(December 2018):1-11.

59. Wang C, Li W, Yin L, Bo J, Peng Y, Wang Y. Comparison of healthy lifestyle behaviors among individuals with and without cardiovascular diseases from urban and rural areas in China: A crosssectional study. 2017;1-13.

60. Fabiyi OO, Garuba OE. Geo-Spatial Analysis of Cardiovascular Disease and Biomedical Risk Factors in Ibadan, South-Western Nigeria. 2018;(July 2015). 
61. Republic of Benin. Republic of Benin - Country Profile. Available from: http://www.nationsonline.org/onewo.

62. Houinato DS, Gbary AR, Houehanou YC, Djrolo F, Amoussou M, Segnon-Agueh J, et al. Prevalence of hypertension and associated risk factors in Benin. Revue d'E'pide'miologie et de Sante'. Publique. 2012;60:95-102.

63. Kandala N. Geographic distribution of cardiovascular comorbidities in South Africa: a national crosssectional analysis. 2014;(March 2015):37-41.

64. Kandala N, Tigbe W, Manda SOM, Stranges S. Geographic Variation of Hypertension in Sub-Saharan Africa: A Case Study of South Africa. Am J Hypertens. 2013;26(March):382-91.

65. Wandai ME, Norris SA, Aagaard-hansen J, Manda SOM. Geographical influence on the distribution of the prevalence of hypertension in South Africa: a multilevel analysis. Cardiovascular Journal of Africa. 2019;30(September 2019):1-8. 